\title{
Evolutionary Psychology's Place in Evolutionary Studies: a Tale of Promise and Challenge
}

\author{
Glenn Geher • Benjamin Crosier • Haley Moss Dillon • \\ Rosemarie Sokol Chang
}

Published online: 12 February 2011

(C) Springer Science+Business Media, LLC 2011

\begin{abstract}
The Evolutionary Studies (EvoS) Consortium and the academic programs born from its creation have been wildly successful in their initial ventures. These achievements are marked by feedback from across the EvoS campuses, the resultant scholarly work produced by participating students, and faculty collaborations spurred by exposure to the organization. The success of EvoS is probably best marked by the recent National Science Foundation grant (CCLI Award \#0817337), awarded jointly to SUNY New Paltz and Binghamton University, with the purpose of expanding EvoS beyond the bounds of these two institutions. A particularly noteworthy element of many EvoS programs is the role of Evolutionary Psychology (EP), a perspective in the behavioral sciences that addresses questions of human behavior from the perspective of evolution. In light of several forms of data, including analyses of a variety of disciplines drawn on from evolutionary psychologists in their work, we argue that evolutionary psychologists may well be the most naturally interdisciplinary scholars within the behavioral sciences, making them highly appropriate for inclusion in EvoS. But our research shows not only promise regarding the relationship between EP and EvoS-challenges are raised as well. We present additional data showing that EP is currently represented disproportionately within the EvoS world - a fact that clearly shows that there are currently limitations to the potential impact of EvoS in modern
\end{abstract}

\footnotetext{
B. Crosier $(\bowtie)$

Department of Psychology, University of Florida,

P.O. Box 112250, Gainesville, FL 32611, USA

e-mail: bencrosier@ufl.edu

G. Geher · H. M. Dillon • R. S. Chang

Department of Psychology, SUNY New Paltz,

New Paltz, NY, USA
}

academia. Scholars from other disciplines, particularly within the humanities and social sciences, seem to be missing the evolution revolution. Implications regarding how EvoS can broaden its scope to be even more powerful in its integrative scope are discussed.

Keywords EvoS · Evolutionary Studies · Evolutionary Psychology · Interdisciplinarity · Citation Analysis

Both evolution itself-along with, more specifically, the application of evolutionary theory to the understanding of human behavior-have had rough-and-tumble histories. This is true inside and outside the Ivory Tower (Wilson et al. 2009). While new theories, methodologies, and ideas in general usually face opposition merely because they are new (Masini 2010), evolution is a charged topic and resistance to the theory comes simultaneously from the general public on religious grounds and from those in academia who feel threatened because biological evolution shakes the foundational assumptions of their discipline (see Geher 2006).

Darwin published his seminal work in 1859 , effectively synthesizing the progress of many biologists before him. The theory has matured largely unscathed; the novelty has long worn off and yet evolution still faces staunch opposition despite a mountain of solid empirical support. These facts result in a number of conditions that make it difficult for the modern teacher and researcher. Regardless of the university, the propagation of such ideas continues to stir controversy, and related research will be attacked and diminished beyond healthy skepticism. Due to the challenges that evolutionary scholars face, it is necessary to actively facilitate such endeavors, both by creating an atmosphere that is conducive to freethinking and by constantly evaluating the growth of the paradigm so the next step is illuminated. 
Further, when people open their minds to the potential of evolution as a framework for illuminating all of lifeincluding humans - significant new insights and facts are uncovered (Wilson 2007). Along with other significant scholars who have emphasized evolution as an explanatory framework across academic areas, David Sloan Wilson (2007) has made the case that evolution (a) is highly applicable to everyday life, (b) is capable of being accurately understood by educated laypeople, and (c) need not be a tool for some evil social agenda. Further, Wilson and his colleagues at Binghamton and New Paltz have demonstrated strongly that Evolutionary Studies (EvoS) has enormous pedagogical benefits for students across a diverse array of academic majors (see Wilson et al. 2009). With these reasons in mind, the Evolutionary Studies Consortium was created.

\section{Successes of the EvoS Consortium}

The integrative power of evolutionary theory has allowed for the EvoS idea to succeed wildly in the landscape of academia since its inception as an undergraduate program at Binghamton in 2003 (Wilson et al. 2009). In thinking about the successes of EvoS, consider the following:

1. EvoS now is represented by over 40 institutions across the world, with the number still growing.

2. The EvoS Seminar Series at New Paltz and Binghamton include some of the most highly visible academic events at these intellectually vibrant institutions. And both schools continue to foster the growth of these serieswhich include speakers on diverse topics from disciplines as diverse as anthropology, biology, geology, literary studies, psychology, and more.

3. Panels on EvoS have been given at such major intellectual venues as the Human Behavior and Evolution Society, the International Society for Human Ethology, and the NorthEastern Evolutionary Psychology Society.

4. The National Science Foundation (NSF) has supported the nationwide expansion of EvoS with $\$ 500,000$ worth of grant monies thus far.

One of the most significant products of EvoS is the EvoS Consortium Website, evostudies.org, which is now a fully functioning, NSF-supported multimedia site that includes a thorough set of information for scholars and students interested in evolutionary studies. This site includes:

1. Information about membership

2. Information about applying for program-starter grants

3. A database of streaming videos from past EvoS speakers

4. A diverse set of blogs dedicated to EvoS

5. Curricular materials to facilitate the teaching of evolutionary concepts
6. Access to the Evolutionary Attitudes and Literacy Survey (developed by Patricia Hawley)

7. A professional-quality newsletter: The EvoS Illuminate

8. A unique academic journal, EvoS Journal: The Journal of the Evolutionary Studies Consortium, that includes both articles about evolutionary studies by scholars across several fields as well as articles related to evolution that are written and peer-reviewed by undergraduate students from across the country (see Chang 2011). This inclusion of undergraduate students at these levels of scholarship is expected to lead a trend in undergraduate training. EvoS Journal is exciting and diverse and is definitely on an upward trajectory.

\section{Successes of Modern-Day Evolutionary Psychology}

In spite of having various conceptions, with slightly different definitions depending on the author, evolutionary psychology, which is essentially the application of evolutionary principles to help inform our understanding of human behavior (see Geher 2006), has met extraordinary success in recent years. Major journals in the behavioral sciences, such as Brain and Behavioral Sciences (see Wilson 2007), and several other major journals (Webster 2007) have embraced evolutionarily informed scholarship in recent years. Further, evolutionary principles have been applied in ways that have shed light on all aspects of psychology, including such basic processes as auditory reception as well as such advanced and complex processes as infanticide and differential parental solicitude (see Barker 2006).

Recently, an additional success of evolutionary psychology has been documented. Compared with other perspectivebased areas of the behavioral sciences, it appears that evolutionary psychology is more interdisciplinary than any other. This point is manifest by the facts that (a) first authors of articles in evolutionary psychology journals are more likely to reflect fields outside psychology compared with journals in other perspective-based areas of psychology and (b) a relatively high number of disciplines outside psychology is cited in journals related to evolutionary psychology compared with journals in other perspective-based areas of psychology (Garcia et al. 2011). In a world where scholarship across disciplines is becoming the ideal, this pattern marks a major new success story regarding evolutionary psychology.

The evolutionary perspective in psychology has come of age - and its presence in modern academia enormously benefits our understanding of factors that underlie human behavior. That said, not everyone loves evolutionary psychology (see McCaughey 2007). In fact, resistance to evolutionary psychology within academia and beyond is significant, palpable, and, ultimately, an issue that simply needs to be addressed for the sake of the future of the field 
(Garcia et al. 2011). Often, evolutionary psychology is framed as an overly deterministic perspective on human behavior that is painted as justifying socially unfair traditions such as sexism (Geher and Gambacorta 2010). While evolutionary psychologists have published many arguments in support of evolutionary psychology, the field is clearly in the midst of a battle with some uphill components (Geher 2006).

\section{Evolutionary Psychology's Place in the World of EvoS}

Evolutionary psychology is a major force in the behavioral sciences with a somewhat unclear, but certainly positive future in academia (Garcia et al. 2011). Further, evolutionary psychology seems to be ahead of the curve when it comes to an interdisciplinary approach to scholarship.

EvoS is, similarly, a growing force in the world of academia - and we believe that the successes of EvoS are still in their infancy. Steeped in the notion of interdisciplinarity, EvoS is a somewhat obvious home for a beast such as evolutionary psychology — particularly given that this beast is not always loved in the world of traditional social science, where major pockets of intellectual resistance to evolutionary psychology remain (Geher and Gambacorta 2010).

But the power of EvoS may be undermined by having evolutionary psychology be disproportionately represented among its ranks. Diversity is a key to the success of EvoS, and a lack of diversity may make EvoS run the risk of becoming conflated with evolutionary psychology in the minds of academics and students.

So the tale here is a bit complex. To the extent that good scholarship is interdisciplinary in nature and sheds light on various kinds of issues, evolutionary psychology is exemplary. In this paper, we summarize novel data that speak to the relatively interdisciplinary nature of evolutionary psychology.

However, this tale has an additional and essential component: While evolutionary psychologists may be doing a great job of reaping the intellectual benefits of evolutionary theory in their work, scholars in many other fields may be lagging considerably behind - a constellation of facts that end up having a significant effect on the composition of EvoS. Two of our subsequent analyses provide evidence for this disproportionate representation of evolutionary psychology in the EvoS Consortium.

\section{Methods}

To address the different questions included in this research, three separate methodologies were utilized. First, a new analysis was conducted to document the relatively interdisciplinary nature of evolutionary psychology relative to other perspective-based areas of psychology. Second, an analysis of the departmental affiliations of individuals who have submitted manuscripts for EvoS Journal was conducted. Similarly, a simple analysis of the departmental affiliations of individuals noted as the university contact persons for the EvoS Consortium was conducted.

Part I: Examining the Tendency for Evolutionary Psychologists to Draw on Disparate Academic Fields

In order to establish the relative interdisciplinarity of the influences in works of evolutionary psychology in comparison to other major fields of psychology, a citation analysis was performed. By identifying the departmental affiliations of the first authors of the works cited in the major journals of these fields, it is possible to highlight the type of research that informs the work contained in each journal. Flagship journals were chosen to represent their respective fields with Evolution and Human Behavior corresponding to evolutionary psychology, Neuroscience embodying the field of neuroscience, and Cognition characterizing cognitive psychology.

Two hundred fifty citations were analyzed for each of the three journals $(N=750)$. Starting with the most recent issue and working backwards, the academic memberships of the first, fifth, tenth ...nth cited authors in journal articles were recorded. Ten citations were acquired from each journal article. Every fifth author was used in order to get an appropriate representation of last names as they are listed alphabetically. Though still not completely random, this is a large improvement from taking the first ten, which would provide only authors with last names that began with the first few letters of the alphabet. This approach helps to eliminate any bias in national or cultural patterns in naming, serving to eliminate an over or under representation of any particular group.

Academic affiliation was classified by the departmental membership of the first author. While this is usually straightforward, as in the example of an author who was listed in an "economics" department and was cataloged as such, some authors were associated with organizations where a decision had to be made, as in "center for disease and public health" being counted as epidemiology. If membership was too ambiguous for the researcher to make an easy call or if the author was affiliated with a nonEnglish speaking institution and no translation could be made, the citation was skipped. Further, sub-disciplines such as social, cognitive, and developmental were considered only under the umbrella term of "psychology." This was, of course, true for non-psychology fields; evolutionary biology, molecular biology, and genetics were all recorded as "biology." While this approach decreases the resolution of gathered information (i.e., it is impossible to tell the exact type 
of science a researcher practices), it speaks more to the questions at hand while simultaneously increasing reliability.

Two coders collected data, evaluating 375 citations each. It was necessary to first establish that an acceptable standard of inter-rater reliability was met before the entire dataset was created. Both investigators initially coded ten citations to establish this criterion. A Cronbach's alpha of 0.846 was achieved for these items, well above the most stringent guideline for acceptable reliability.

Part II: Content Analysis of Disciplines Represented in EvoS Journal

To maintain confidentiality, the editor of EvoS Journal (RSC) performed this portion of the analysis. For both student and faculty authors, the academic affiliations were recorded. No identifying information, save departmental membership, was used in this analysis. To conform with part I, only affiliations of the first authors are included in this analysis.

Part III: Content Analysis of Disciplines Represented in EvoS Membership

Given that 42 institutions now have some level of membership in the EvoS Consortium, the departmental affiliations of the contact person for each of the EvoS programs were analyzed to see if Psychology is disproportionately represented in terms of this data source. The affiliations were collected from the data that comprise the evostudies.org membership database.

\section{Results}

Part 1: Examining the Tendency for Evolutionary Psychologists to Draw on Disparate Academic Fields

The first citation analysis revealed that these specific toptier journals do disproportionately cite work from psychology at a relatively high frequency $\left(\chi^{2}(2, N=750)=87.597\right.$, $p<0.05)$. While the sampled papers in Evolution and Human Behavior cited 85 psychology references and Neuroscience cited 78, Cognition drew from 209 works of psychology, tipping the balance. The historically older field of cognitive psychology tended to stay more in line with its theoretical boundaries, while the historically recent fields tended to reach beyond psychology. Next, speaking to its relatively high level of interdisciplinarity, Evolution and Human Behavior displayed that it was represented by a remarkably more diverse body of cited literature, drawing from nearly twice as many fields as Cognition and nearly three times as many fields as Neuroscience (28, 16, and 9 disciplines, respectively; $\left.\chi^{2}(2, N=750)=21.906, p<0.001\right)$. In sum, the application of evolution to human behavior has no more of a psychological influence than any other subfield of psychology, yet it is incredibly strong in terms of interdisciplinarity.

Part II: Content Analysis of Disciplines Represented in EvoS Journal

Twenty-one papers have been submitted to EvoS Journal since it was formed in 2009. The first authors are from the following departments: psychology (17), biology (3), and anthropology (1). A chi-square analysis revealed that there are significantly more submissions from psychology departments than the other two departments $\left(\chi^{2}(1, N=21)=8.047\right.$, $p=0.005$ ).

Part III: Content Analysis of Disciplines Represented in EvoS Membership

There are 35 colleges and universities listed as EvoS affiliates on the consortium website and six non-college and universities (e.g., societies, museums). For the purposes of this analysis, we tested only the colleges and universities. Of these, the contacts listed came mostly from three departments (17 from biology, 9 from psychology, and 4 from anthropology), and one each came from five different departments, which have been collapsed into one miscellaneous category.

There is a significant difference in the representation of departments $\left(\chi^{2}(3, N=35)=11.97, p=0.008\right)$. But when comparing the two most frequent departments, psychology and biology, there is not a significant difference in the representation of each $\left(\chi^{2}(1, N=26)=2.46, p=0.116\right)$.

\section{Conclusion}

The core players in EvoS have written several pieces emphasizing the success of the EvoS idea (e.g., Carmen et al. 2010; Chang et al. 2009; Geher and Gambacorta 2010; Wilson et al. 2009; Wilson 2007). Given the powerful nature of evolutionary theory at providing insights into phenomena near and far and the support of the home institutions of the initial EvoS programs, along with support of the NSF, the early successes of the EvoS Consortium make good sense.

That said, a major long-term goal of EvoS is to have EvoS programs integrated broadly into college curriculaideally, we envision a college's EvoS program being as fundamental as its biology, psychology, or sociology departments. Clearly, that's a big goal that will take time to realize. And, as is evident across the articles in this 
special issue of Evolution: Education and Outreach, we're working on it!

Evolutionary psychology is an exceptional area of psychology with a major impact both within the academy (see Wilson 2007) and within modern culture more broadly (see Fisher et al. 2011). But evolutionary psychology is not perfect. And it has some public relations issues to take care of to facilitate its positive growth in the future (Garcia et al. 2011).

Our analyses here dovetail strongly with prior research showing that evolutionary psychology represents a relatively interdisciplinary endeavor, conceptually replicating the work of Garcia et al. (2011). Evolutionary psychologists cite literature from diverse areas in their work-with considerably more diversity compared with the diversity of literature cited by psychologists in other perspective-based areas (such as psychodynamics or cognitive psychology). A relatively interdisciplinary approach such as evolutionary psychology makes good sense in an academic home that embraces interdisciplinary scholarship - and EvoS is clearly such a home. And a successful, exciting home with lots of growth opportunities, at that.

So it seems that EvoS is very good for evolutionary psychology. But in thinking about the broad goals of the EvoS Consortium, we wonder if the relationship is fully mutually beneficial. While the contributors to Evolution and Human Behavior, a top journal in evolutionary approaches to behavior (Garcia et al. 2011), are representative of many disciplines, EvoS Journal to date draws interest primarily from psychologists. EvoS Journal would benefit by continuing to reach in directions other than psychology. EvoS is premised on an interdisciplinary approach. To explicate this point, it is worth considering the fact that one of the truly exciting elements of the EvoS Seminar Series at both New Paltz and Binghamton pertains to the diversity in the background of the speakers. In recent years, New Paltz has hosted speakers from anthropology, biology, geology, philosophy, and psychology. Binghamton's EvoS Series represents even more disciplines (including economics and history). Seeing how evolutionary principles connect these different areas of scholarship on various topics is the essence of the EvoS experience.

Our analyses demonstrate in part that evolutionary psychology is disproportionately represented in EvoS. Though in our analysis the finding was not significant, that biologists in absolute terms outnumber psychologists as contacts for consortium-interested institutions is reflective of a few things. The first is that the main proponent of EvoS, David Sloan Wilson, is a biologist and thus presumably has more contacts within that field than any other. The second is that we have restricted in our category of psychologists people who are in psychology departments, though people publishing in the realm of evolutionary psychology are by no means limited to this field. The outcome that more first authors for EvoS Journal submissions are from psychology departments is reflective of the interest we receive from sign-ups after presenting EvoS at international conferences. At a recent interdisciplinary conference, $56 \%$ of people who signed up for the listserv are psychologists.

Contrary to what some might think, however, we don't think evolutionary psychologists are going down a dead-end! Further, we don't think that EvoS is doing anything wrong! The problem presented here is a developmental problem, and it reflects the current states of both EvoS and evolutionary psychology in modern academia.

Think of Shrek and Donkey - two formidable characters, each with plenty of positive attributes. When joined together as a team, this pair is capable of doing anything. When divided, they can become stagnant, non-productive, and even bitter. The relationship between evolutionary psychology and EvoS is similar - and for EvoS to be its best - which means being representative of varied disciplines - can help evolutionary psychology be its best, which means thriving in an interdisciplinary castle!

\section{Limitations and Future Research}

While this research suggests that evolutionary psychology is a relatively interdisciplinary area of scholarship and that evolutionary psychologists are slightly over-represented among the EvoS consortium (certainly in terms of contributing to EvoS Journal), there are some methodological caveats that are important to consider. For one, there may be issues concerning the validity of our measure of interdisciplinarity. To come up with an algorithm that could be consistently employed, we chose the affiliations of first authors of articles in the journals sampled. Heterogeneity among affiliations within author-groups may certainly exist - and may provide an additional marker of interdisciplinarity. And other possible algorithms are possible. Future research could explore these different kinds of algorithms for operationally defining interdisciplinarity to move toward a relatively valid index.

Also, the current analyses bear explicitly on psychologynot addressing whether, for instance, evolutionary biology is more interdisciplinary than other areas of biology, etc. This is an important question for addressing if the interdisciplinarity that follows an evolutionary approach in psychology tends to follow from evolutionary approaches employed in other fields.

Given the student-oriented nature of EvoS, markers of interdisciplinarity that follow student interests may be useful to analyze as well. A recent analysis of one 20person section of Evolutionary Studies Seminar (EVO 301) taught at SUNY New Paltz revealed 11 different majors among the 20 students - this is clearly an interdisciplinary 
group. More systematic, targeted research along these lines would be very informative in terms of how interdisciplinary EvoS is at the level of the student curricular experience.

Additionally, the diversity of academic backgrounds represented in one of our EvoS seminar series may serve as a useful marker of interdisciplinarity. Consider, for instance, the fact that in the 2011 Spring EvoS Seminar at New Paltz, fields represented include Anthropology, Biology, English, Psychology, and Social Work-quite a spread-and this diversity is typical of the different EvoS Series that exist. Again, more comprehensive and systematic research on this topic would be empirically beneficial.

One of our main points was that EvoS is over-represented by psychologists. This hypothesis was only partially supported. Based on the affiliations of the primary contact individuals for the different programs in the EvoS Consortium, it seems that biology is the most-represented field in EvoS. But based on the analysis of author affiliation of EvoS Journal, it seems that psychologists are disproportionately represented as authors in that journal. So regarding the question of whether psychology is overly represented in EvoS, the answer is "it depends how you look at it."

Further, there are some methodological complications regarding the analysis of departmental affiliation and authorship in EvoS Journal. This inference is based on affiliations as psychologists, not "evolutionary psychologists" per se. It may actually be the case that this journal is drawing in psychology authors who are not aligned with an evolutionary perspective. Future research would be needed to tease this issue apart-but it's an intriguing idea as it may imply that EvoS Journal is perceived by non-evolutionary psychologists as a sort of safe, neutral territory.

\section{Bottom Line}

Consistent with David Sloan Wilson's (2007) call for scholars and students of all shapes and sizes to learn how to employ evolutionary principles to facilitate productive scholarship, we think that the main issue here is that evolution simply has not seeped sufficiently into the fabric of most academic disciplines. To help reach its goals of further expansion, we believe that EvoS needs to more explicitly reach out to those disciplines that are currently naive to the power and wonder of the theory of evolution.
The success of EvoS' future depends significantly on these disciplines that have so far remained disconnected from EvoS in the world of the Ivory Archipelago. Realizing this issue and coming up with a plan to address it should benefit the EvoS Consortium and, we believe, should ultimately benefit our understanding of phenomena near and far.

\section{References}

Barker L. Teaching evolutionary psychology: an interview with David M. Buss. Teach Psychol. 2006;33:69-76.

Carmen R, Moss Dillon H, Geher G. History, biology, and politics neatly intertwined: Lee Dugatkin's newest work as an exemplar of an EvoS education. EvoS Journal: The Journal of the Evolutionary Studies Consortium. 2010;2:67-71.

Chang RI, Geher G, Waldo J, Wilson DS. EvoS for everyone: The launch of the EvoS journal. EvoS Journal: The Journal of the Evolutionary Studies Consortium. 2009;1:1-2.

Chang RS. Passing the red pen: EvoS Journal offers an early education in peer review. Evolution:Education and Outreach. 2011;4pp.

Fisher ML, Kruger DJ, Garcia JR. Understanding and enhancing the role of mass media in evolutionary psychology education. Evolution: Education and Outreach. 2011;4pp.

Garcia J, Geher G, Crosier B, Saad G, Gambacorta D, Johnsen L, et al. The interdisciplinarity of evolutionary approaches to human behavior: a key to survival in the Ivory Archipelago. Futures. 2011 ; in press.

Geher G. Evolutionary psychology is not evil! ... and here's why... Psychological Topics. 2006;15:181-202.

Geher G, Gambacorta D. Evolution is not relevant to sex differences in humans because I want it that way! Evidence for the politicization of human evolutionary psychology. EvoS Journal: The Journal of the Evolutionary Studies Consortium. 2010;2:3247.

Masini EB. New challenges for futures studies. Futures. 2010;33:63747.

McCaughey M. The caveman mystique: Pop-Darwinism and the debates over sex, violence, and science. New York: Routledge; 2007.

O'Brien DT, Wilson DS, Hawley PH. "Evolution for everyone": a course that expands evolutionary theory beyond the biological sciences. Evol Educ Outreach. 2009;2:445-57.

Webster GD. Evolutionary theory in cognitive neuroscience: a 20 -year quantitative review of publication trends. Evol Psychol. 2007;5:520-30.

Wilson DS. Evolution for everyone. New York: Delacorte; 2007.

Wilson DS, Geher G, Waldo J. EvoS: completing the evolutionary synthesis in higher education. EvoS J J Evol Stud Consortium. 2009;1:3-10. 